\title{
Supplemental Results
}

\section{Genetic and Environmental Models of Executive Function, Verbal Fluency, and Other \\ Cognitive Abilities}

Adolescents. After adding vocabulary and nonverbal cognitive ability into the genetic model, the unique genetic variance components remained significant for the General Fluency factor, explaining 38\% of its total variance, $95 \%$ CI $[.10, .51]$, but not for the Semantic-Specific factor, $31 \%, 95 \%$ CI $[.00, .57]$. In this final model with EFs, vocabulary, and nonverbal cognitive ability, there is a further reduction in power to detect unique genetic influences on the fluency factors, especially Semantic-Specific. As the number of variables in the model increases, there are more places for residual genetic influences to move around when fixed to zero, especially because both vocabulary and nonverbal cognitive ability have substantial genetic overlap with the EF factors and each other (and therefore smaller unique genetic influences themselves). Similar results are observed for the corresponding analyses in middle age (below). Thus, rather than interpret the nonsignificant difference tests as a lack of evidence for unique genetic influences, we focus on the size of estimates themselves (31 to 38\%), and leverage the phenotypic regression analyses where the total residual variance was highly significant on both fluency factors at both ages.

Middle-aged adults. After adding vocabulary and nonverbal cognitive ability into the model, unique genetic influences on the General Fluency factor (explaining 22\% of its total variance, $95 \%$ CI $[.00, .42])$ and the Semantic-Specific factor $(53 \%, 95 \%$ CI $[.00, .76])$ were substantial but nonsignificant.

\section{Additional Phenotypic Analyses Involving Perceptual and Processing Speed}

Adolescents. We repeated phenotypic multiple regression analyses after including a Perceptual Speed latent factor based on the one used by Friedman et al. (2008) with the Hidden Patterns and Identical Pictures tests (Ekstrom, French, Harman, \& Derman, 1976). Previous work also included a third Colorado perceptual speed task (Defries, Plomin, Vandenberg, \& Kuse, 1981), but this was excluded because of its verbal and working memory demands. Including this third task would not affect the results 
shown below, but a model including it had worse RMSEA and CFI values, and had slightly inflated correlations between the Perceptual Speed factor and the General Fluency, Vocabulary, and Working Memory factors unless including additional factor loadings from this task to these latent factors.

Results of these additional analyses are displayed in Table S4 (correlations) and Table S5 (multiple regression). Importantly, although the Perceptual Speed factor was correlated with all other cognitive abilities (except Shifting-Specific ability) and remained significant in the multiple regression model, the associations between General Fluency and Common EF, Shifting-Specific, and UpdatingSpecific factors remained significant. The $\mathrm{R}^{2}$ value of .52 in this model was similar to that in the main text without controlling for perceptual speed (.50). Similar results were observed for the SemanticSpecific factor. The Semantic-Specific factor was still associated with the Shifting-Specific factor after controlling for perceptual speed, with over half the variance still unaccounted for by all other cognitive abilities $\left(\mathrm{R}^{2}=.48\right)$. After controlling for the Perceptual Speed factor, there was a negative association between the Semantic-Specific and Common EF factors, but we do not interpret this result given that the overall correlation was nonsignificant $(r=-.03)$

Middle-aged adults. In the middle-aged sample, we assessed processing speed by including a latent Reaction Time factor previously described by Gustavson et al. (2018). This factor was based on two computerized tasks: a simple RT task and a two-choice RT task. Again, although Reaction Time was associated with most other cognitive abilities, the results of the regression analyses were largely identical. The General Fluency factor remained associated with the Common EF, WM-Specific, and Vocabulary factors and the Semantic-Specific factor remained associated with the Vocabulary factor. The Reaction Time factor was only weakly associated with the Semantic-Specific factor, and the $\mathrm{R}^{2}$ values were nearly identical to those in the analyses without reaction time.

Summary and discussion. These results suggest that the associations between EFs and verbal fluency were not explained by processing speed, and that processing speed can only account for a small portion of the residual variance in verbal fluency factors. Important to note, the speed measures were very different in each sample. The Perceptual Speed factor was much more strongly correlated with EFs and 
Vocabulary in adolescents (even excluding the third task that had the strongest verbal demands), compared to correlations with the Reaction Time factor in middle-aged adults. This pattern likely reflects the complexity of the perceptual speed tasks assessed in the adolescent sample, compared to the reaction time tasks assessed in middle-aged adults (which we view as a much purer measure of speed of processing). Thus, even though there was some reduction in the association between the Common EF and General Fluency factors after controlling for Perceptual Speed $(\beta=.32$ versus .27$)$ in the adolescent sample, it is unlikely that processing speed accounts for a large portion of the covariance between fluency and EFs in either sample, and it is clear that there is considerable variance remaining in fluency not explained by the cognitive abilities assessed here. 
Table S1

Phenotypic Correlations Among All Measures of the Study in the Adolescent Sample (CLTS - Mean Age 17)

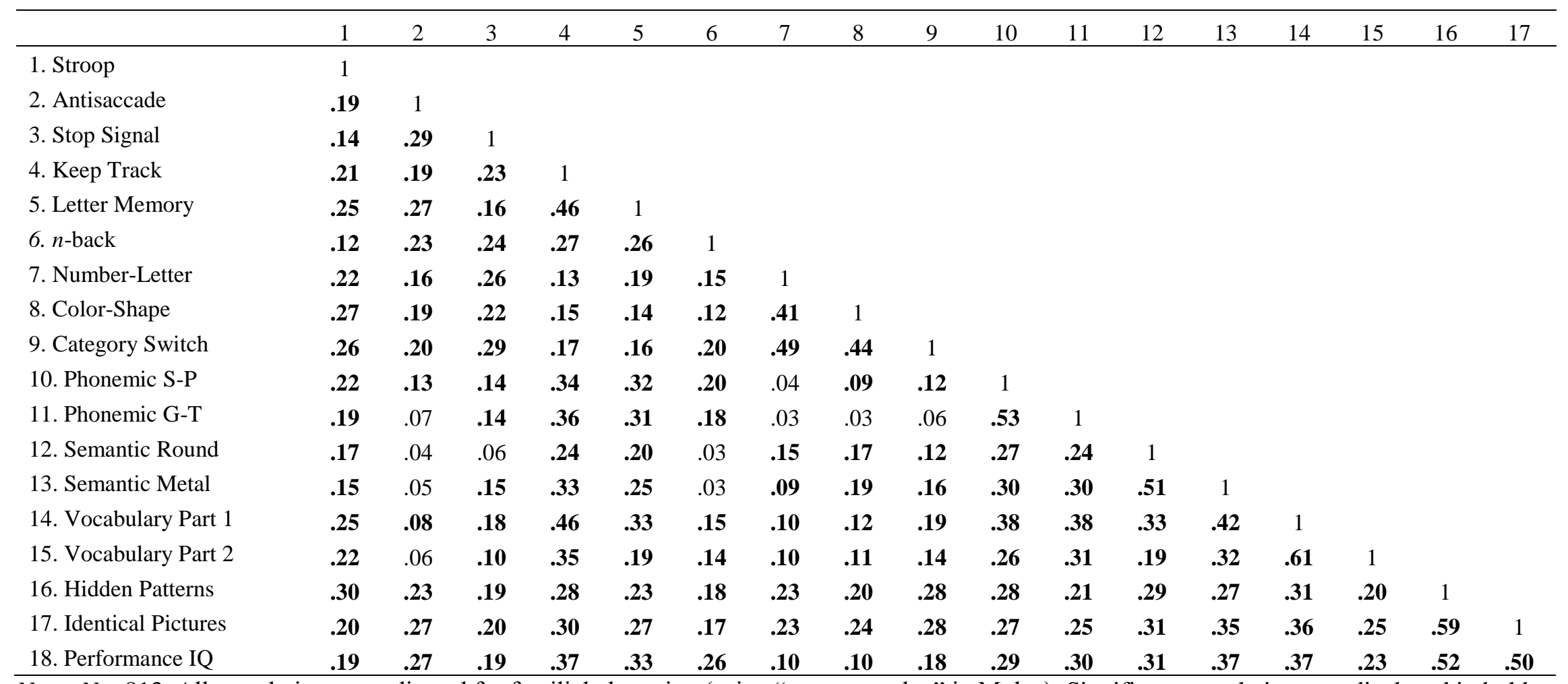

Note: $N=813$. All correlations are adjusted for familial clustering (using "type=complex" in Mplus). Significant correlations are displayed in bold $(p<.05)$. 
Table 52

Phenotypic Correlations Among All Measures of the Study in the Middle-Aged Sample (VETSA - Mean Age 56)

\begin{tabular}{|c|c|c|c|c|c|c|c|c|c|c|c|c|c|c|c|c|c|}
\hline & 1 & 2 & 3 & 4 & 5 & 6 & 7 & 8 & 9 & 10 & 11 & 12 & 13 & 14 & 15 & 16 & 17 \\
\hline 1. Stroop & 1 & & & & & & & & & & & & & & & & \\
\hline 2. AX-CPT & .16 & 1 & & & & & & & & & & & & & & & \\
\hline 3. Trail Making Test & .16 & .27 & 1 & & & & & & & & & & & & & & \\
\hline 5. Reading Span & .15 & .21 & .18 & .43 & 1 & & & & & & & & & & & & \\
\hline 6. Digit Span & .14 & .18 & .26 & .59 & .51 & 1 & & & & & & & & & & & \\
\hline 7. Phonemic F & .08 & .18 & .12 & .28 & .26 & .32 & 1 & & & & & & & & & & \\
\hline 9. Phonemic S & .07 & .13 & .14 & .28 & .26 & .31 & .62 & .61 & 1 & & & & & & & & \\
\hline 10. Animals & .08 & .15 & .13 & .20 & .24 & .18 & .33 & .31 & .36 & 1 & & & & & & & \\
\hline 11. Boys' Names & .08 & .15 & .14 & .25 & .28 & .27 & .38 & .44 & .44 & .42 & 1 & & & & & & \\
\hline 12. Fruits/Furniture & .09 & .17 & .17 & .23 & .22 & .20 & .25 & .30 & .28 & .34 & .31 & 1 & & & & & \\
\hline 13. WASI - Vocabulary & .17 & .26 & .19 & .39 & .47 & .41 & .31 & .38 & .33 & .31 & .28 & .23 & 1 & & & & \\
\hline 14. AFQT - Vocabulary & .15 & .25 & .24 & .32 & .36 & .35 & .22 & .29 & .24 & .22 & .19 & .17 & .58 & 1 & & & \\
\hline 18. AFQT - Nonverbal & .15 & .22 & .21 & .22 & .25 & .22 & .13 & .15 & .15 & .19 & .14 & .18 & .37 & .33 & .08 & .11 & .46 \\
\hline
\end{tabular}

Note: $N=1290$. All correlations are adjusted for familial clustering (using "type=complex" in Mplus). AX-CPT = AX - Continuous Performance Test; WASI = Wechsler Abbreviated Scale of Intelligence; AFQT = Armed Forced Qualification Test; RT = Reaction Time. Significant correlations are displayed in bold $(p<.05)$. 
Table S3: Factor Loadings for all Tasks in the Phenotypic Models (Tables 2 and 3)

\begin{tabular}{|c|c|c|c|c|c|c|c|c|}
\hline Task & $\begin{array}{l}\text { Common } \\
\text { EF }\end{array}$ & $\begin{array}{l}\text { Updating } \\
\text { /WM- } \\
\text { Specific }\end{array}$ & $\begin{array}{l}\text { Shifting- } \\
\text { Specific }\end{array}$ & $\begin{array}{l}\text { General } \\
\text { Fluency }\end{array}$ & $\begin{array}{l}\text { Semantic- } \\
\text { Specific }\end{array}$ & Vocabulary & Speed & $\begin{array}{l}\text { Nonverbal } \\
\text { Intelligence }\end{array}$ \\
\hline \multicolumn{9}{|l|}{ Age 17 (CLTS) } \\
\hline Stroop & 0.42 & - & - & - & - & - & - & - \\
\hline Antisaccade & 0.51 & - & - & - & - & - & - & - \\
\hline Stop Signal & 0.47 & - & - & - & - & - & - & - \\
\hline Keep Track & 0.41 & 0.65 & - & - & - & - & - & - \\
\hline Letter Memory & 0.44 & 0.44 & - & - & - & - & - & - \\
\hline$n$-back & 0.44 & 0.12 & - & - & - & - & - & - \\
\hline Number-Letter & 0.42 & - & 0.52 & - & - & - & - & - \\
\hline Color-Shape & 0.38 & - & 0.51 & - & - & - & - & - \\
\hline Category Switch & 0.50 & - & 0.50 & - & - & - & - & - \\
\hline Phonemic S-P & - & - & - & 0.73 & - & - & - & - \\
\hline Phonemic G-T & - & - & - & 0.73 & - & - & - & - \\
\hline Semantic Round & - & - & - & 0.20 & 0.58 & - & - & - \\
\hline Semantic Metal & - & - & - & 0.26 & 0.67 & - & - & - \\
\hline Vocabulary Part 1 & - & - & - & - & - & 0.93 & - & - \\
\hline Vocabulary Part 2 & - & - & - & - & - & 0.66 & - & - \\
\hline Hidden Patterns & - & - & - & - & - & - & 0.76 & - \\
\hline Identical Pictures & - & - & - & - & - & - & 0.77 & - \\
\hline Performance IQ & - & - & - & - & - & - & - & 1 \\
\hline \multicolumn{9}{|l|}{ Age 56 (VETSA) } \\
\hline Stroop & 0.32 & - & - & - & - & - & - & - \\
\hline $\mathrm{AX}-\mathrm{CPT}$ & 0.53 & - & - & - & - & - & - & - \\
\hline Trail Making Test & 0.49 & - & - & - & - & - & - & - \\
\hline Letter-Number & 0.49 & 0.51 & - & - & - & - & - & - \\
\hline Reading Span & 0.23 & 0.35 & - & - & - & 0.29 & - & - \\
\hline Digit Span & 0.42 & 0.74 & - & - & - & - & - & - \\
\hline Phonemic F & - & - & - & 0.77 & - & - & - & - \\
\hline Phonemic A & - & - & - & 0.78 & - & - & - & - \\
\hline Phonemic S & - & - & - & 0.80 & - & - & - & - \\
\hline Animals & - & - & - & 0.43 & 0.63 & - & - & - \\
\hline Boys' Names & - & - & - & 0.54 & 0.31 & - & - & - \\
\hline Fruits/Furniture & 0.16 & - & - & 0.29 & 0.28 & - & - & - \\
\hline WASI - Vocab. & - & - & - & - & - & 0.84 & - & - \\
\hline AFQT - Vocab. & - & - & - & - & - & 0.69 & - & - \\
\hline Simple RT & - & - & - & - & - & - & 0.71 & - \\
\hline Choice RT & - & - & - & - & - & - & 0.93 & - \\
\hline WASI - Matrix & - & - & - & - & - & - & - & 0.74 \\
\hline AFQT - Nonverbal & - & - & - & - & - & - & - & 0.61 \\
\hline
\end{tabular}

Note: The second column refers to the Updating-Specific factor in adolescence (age 17), but the WMSpecific factor in middle age (age 56). All factor loadings are statistically significant $(p<.05)$, with the exception of $n$-back on Updating-Specific, $\chi_{\text {diff }}^{2}(1)=2.28, p=.131$. 


\section{Table S4}

Latent Variables Correlations between Verbal Fluency, Executive Functions, Vocabulary, Processing Speed, and Nonverbal Cognitive Ability

\begin{tabular}{|c|c|c|c|c|c|c|c|}
\hline & $\begin{array}{l}\text { General } \\
\text { Fluency }\end{array}$ & $\begin{array}{l}\text { Semantic- } \\
\text { Specific }\end{array}$ & $\begin{array}{c}\text { Common } \\
\text { EF }\end{array}$ & $\begin{array}{l}\text { Updating- } \\
\text { Specific }\end{array}$ & $\begin{array}{l}\text { Shifting- } \\
\text { Specific }\end{array}$ & Vocabulary & Speed \\
\hline \multicolumn{8}{|l|}{ Age 17} \\
\hline General Fluency & 1 & & & & & & \\
\hline Semantic-Specific & - & 1 & & & & & \\
\hline Common EF & 0.42 & -0.03 & 1 & & & & \\
\hline Updating-Specific & 0.49 & 0.28 & - & 1 & & & \\
\hline Shifting-Specific & -0.17 & 0.39 & - & - & 1 & & \\
\hline Vocabulary & 0.56 & 0.32 & 0.32 & 0.54 & 0.05 & 1 & \\
\hline Perceptual Speed & 0.46 & 0.37 & 0.58 & 0.20 & 0.14 & 0.47 & 1 \\
\hline \multirow[t]{2}{*}{ Nonverbal Cognitive Ability } & 0.40 & 0.31 & 0.48 & 0.27 & -0.14 & 0.39 & 0.66 \\
\hline & $\begin{array}{l}\text { General } \\
\text { Fluency }\end{array}$ & $\begin{array}{l}\text { Semantic- } \\
\text { Specific }\end{array}$ & $\begin{array}{c}\text { Common } \\
\text { EF }\end{array}$ & $\begin{array}{c}\text { WM- } \\
\text { Specific }\end{array}$ & & Vocabulary & Speed \\
\hline \multicolumn{8}{|l|}{ Age 56} \\
\hline General Fluency & 1 & & & & & & \\
\hline Semantic-Specific & - & 1 & & & & & \\
\hline Common EF & 0.40 & 0.18 & 1 & & & & \\
\hline WM-Specific & 0.33 & -0.04 & - & 1 & & & \\
\hline Vocabulary & $\mathbf{0 . 5 0}$ & 0.22 & 0.58 & 0.34 & - & 1 & \\
\hline Reaction Time & 0.19 & 0.15 & 0.26 & 0.07 & - & 0.11 & 1 \\
\hline Nonverbal Cognitive Ability & 0.35 & 0.25 & 0.72 & 0.11 & - & $\mathbf{0 . 7 0}$ & 0.13 \\
\hline
\end{tabular}

Note: Significant correlations are displayed in bold $(p<.05)$. All constructs were measured with latent variables except nonverbal cognitive ability in adolescents (i.e., WAIS performance IQ). Models fit the data well at both waves, $\chi^{2}(104)=228.69, p<.001$, RMSEA=.038 for adolescents, $\chi^{2}(108)=176.80, p<.001$, RMSEA $=.022$ for middle-aged adults. 


\section{Table S5}

Regression Analyses Involving Executive Functions, Vocabulary, Processing Speed, and Nonverbal Cognitive Ability

\begin{tabular}{|c|c|c|c|c|c|c|c|}
\hline & $\begin{array}{l}\text { Common } \\
\text { EF }\end{array}$ & $\begin{array}{l}\text { Updating/WM- } \\
\text { Specific }^{\text {a }}\end{array}$ & $\begin{array}{l}\text { Shifting- } \\
\text { Specific }\end{array}$ & Vocabulary & Speed & $\begin{array}{c}\text { Nonverbal } \\
\text { Cognitive } \\
\text { Ability }\end{array}$ & $\mathrm{R}^{2}$ \\
\hline \multicolumn{8}{|l|}{ Age 17} \\
\hline General Fluency & 0.27 & 0.34 & -0.22 & 0.24 & 0.20 & -0.07 & 0.52 \\
\hline Semantic-Specific & -0.28 & 0.15 & 0.32 & 0.20 & 0.29 & 0.27 & 0.48 \\
\hline \multicolumn{8}{|l|}{ Age 56} \\
\hline General Fluency & 0.27 & 0.22 & - & 0.36 & 0.08 & -0.13 & 0.33 \\
\hline Semantic-Specific & -0.06 & -0.09 & - & 0.20 & 0.14 & 0.19 & 0.13 \\
\hline
\end{tabular}

Note: This table displays the same multiple regression results as those from Table 4 of the main text, except including another latent independent variable for perceptual speed (age 17) or reaction time (age 56). Fluency factors were included as dependent variables with correlations among all independent variables (EF factors, vocabulary, speed, nonverbal cognitive ability), except correlations among EF factors were fixed to zero. Significant regression coefficients are displayed in bold $(p<.05)$. Correlations among independent variables were identical to those from the correlational models (Table S4). All constructs were measured with latent variables except nonverbal cognitive ability in adolescents (i.e., WAIS performance IQ). ${ }^{\text {a }}$ indicates Updating-Specific at age 17 but Working Memory-Specific at age 56. 

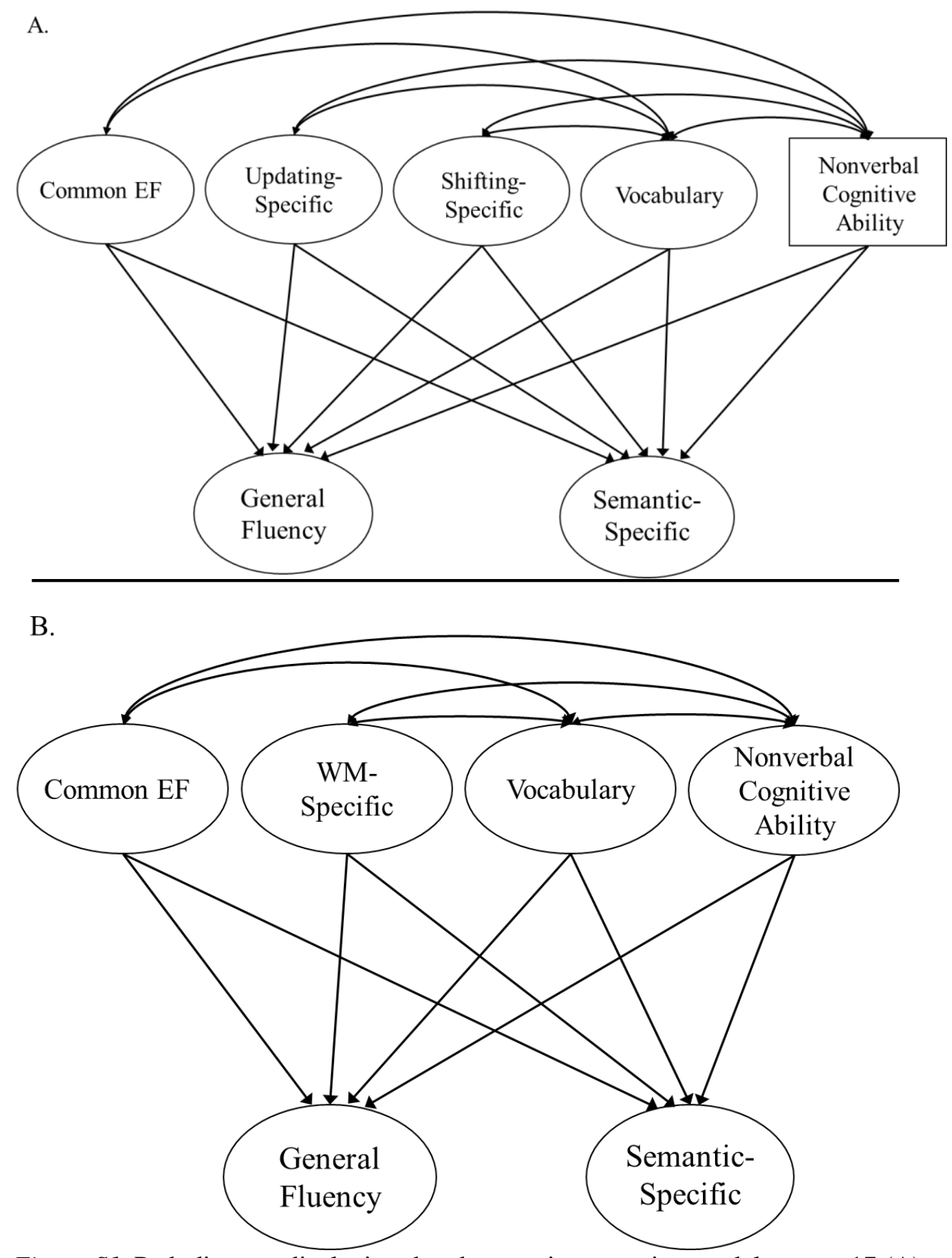

Figure S1. Path diagram displaying the phenotypic regression models at age 17 (A) and age 56 (B) from Table 3. Factor loadings from latent variables to individual tasks are not displayed, but are shown in Table S1. Ovals indicate latent variables and rectangles indicate measured variables (Nonverbal cognitive ability was assessed at age 17 with the WAIS performance IQ score). 


\section{References}

Defries, J. C., Plomin, R., Vandenberg, S. G., \& Kuse, A. R. (1981). Parent-offspring resemblance for cognitive-abilities in the Colorado Adoption Project: Biological, adoptive, and control parents and one-year-old children. Intelligence, 5, 245-277. doi:10.1016/S0160-2896(81)80012-8

Ekstrom, R. B., French, J. W., Harman, H. H., \& Derman, D. D. (1976). Kit of factor-referenced cognitive tests. Princeton, NJ: Educational Testing Service.

Friedman, N. P., Miyake, A., Young, S. E., Defries, J. C., Corley, R. P., \& Hewitt, J. K. (2008). Individual differences in executive functions are almost entirely genetic in origin. Journal of Experimental Psychology: General, 137, 201-225. doi:10.1037/0096-3445.137.2.201

Gustavson, D. E., Panizzon, M. S., Franz, C. E., Friedman, N. P., Reynolds, C. A., Jacobson, K. C., . . . Kremen, W. S. (2018). Genetic and environmental architecture of executive functions in midlife. Neuropsychology, 32, 18-30. doi:10.1037/neu0000389 